\section{Internal iliac artery ligation - A life saving procedure in Obstetrics and Gynaecology practice}

\author{
Prof (Dr) Hemkanta Sarma
}

It was way back in 1894 when Sir Howard Kelly performed bilateral iliac artery ligation to control intraoperative pelvic haemorrhage for cervical cancer for the first time. It had not been used extensively in the appropriate moment ${ }^{1}$. As a result many a time the reproductive life of a young woman had to be compromised. Infact this had even resulted in the unfortunate loss of lives. This is one of the most effective methods of controlling post partum haemorrhage ${ }^{1-3}$.

Postpartum haemorrhage (PPH) is one of the major causes of maternal death worldwide ranging from $13 \%$ in developed countries to $34 \%$ in the developing countries ${ }^{1}$. As modern medicine progresses there have been many more advances in the treatment of obstetric haemorrhages, gynaecological postoperative haemorrhages and coagulation disorders. At the advent of the modern gadgets like MRI, CT scan etc., selective embolization of pelvic vessels is also playing a great role in this regard. Even then this procedure of internal iliac artery ligation has its own importance in controlling pelvic haemorrhage -whether it is intraoperative or postoperative, whether it is of infective origin or due to erosion of vessels by malignant infiltration. All obstetricians and gynaecologists, specially those who deal with critical care obstetrics should learn the technique of internal iliac ligation which is not difficult, provided the surgeon has good knowledge of pelvic anatomy.

\section{Mechanism of Haemostasis}

Following bilateral iliac artery ligation, there is fall of pulse pressure, mean arterial pressure, and blood flow which promotes the clot formation. The ligation converts

\author{
Correspondence: \\ Prof (Dr) Hemkanta Sarma, \\ Professor \& HOD, Department \\ of Obstetrics and Gynaecology, \\ Jorhat Medical College, Jorhat, \\ Assam, India; Email - \\ sarmahemkanta@gmail.com

\section{Distributed under Creative Commons Attribution-Share Alike 4.0 International.}

the arterial system into venous system leading to stable clot formation, thus bringing haemostasis. It has been observed that pulse pressure is reduced by $77 \%$ with unilateral ligation and $85 \%$ with bilateral ligation (Clark 1985). The rate of blood flow dropped upto $48 \%$ on the same side after ligation of the internal iliac artery ${ }^{1}$.

\section{Collateral Circulation}

A good collateral circulation is established after the bilateral internal iliac artery ligation (BIIAL). Out of all, the vertical or ipsilateral collaterals are more effective. These are as follows -

1. Lumbar vessels (branch of aorta) with iliolumbar vessels (branch of internal iliac).

2. Middle sacral vessels (branch of aorta) with lateral sacral vessels (branch of internal iliac).

3. Superior haemorrhoidal (branch of inferior mesenteric) with middle haemorrhoidal (branch of internal iliac).

All these collaterals start functioning immediately after the ligation. There is ample evidence in the literature of good collateral circulation supporting the uterus in healthy state leading to successful pregnancy following bilateral internal iliac ligation. In an unusual case of PPH following caesarean section, the author has noticed recurrence of vaginal bleeding following BIIAL after 2 weeks when hysterectomy was performed to save the patient.

\section{Indications}

Any haemorrhage from a pelvic organ which is supplied by the arteries arising from the anterior division of internal iliac artery may be controlled by the BIIAL . 
The common examples are ${ }^{4}$

1. Uterine atony ( $\mathrm{PPH})$

2. Uterine rupture

3. Placental morbid adhesion (eg. Accreta, Increta, Percreta ).

4. Radical hysterectomy.

5. Post operative haemorrhage (primary / secondary).

6. Anticipated operative bleeding.

7. Massive pelvic trauma.

8. Pelvic malignancy leading to bleeding due to vessel erosion.

\section{Approaches}

1) Intra peritoneal: This is less time consuming conventional method. It is mostly useful during emergency situation - usually intraoperative.

2) Extra peritoneal: This method is useful in planned elective cases. It is more relevant for immediate postoperative cases and especially in those cases that has intraperitoneal infection or has oedematous, friable tissues due to recent surgical attempts.

3) Modified extraperitoneal technique: This method has been innovated and used by the author in 15 cases in different institutions of Silchar, Guwahati and Barpeta while dealing with emergency situations of uncontrolled pelvic haemorrhages. This method is useful in postoperative cases where pfannenstiel incision was made and extraperitoneal technique of BIIAL is decided. In this method, instead of opening the peritoneal cavity, or making two different suprainguinal incisions, the original pfannenstiel incision is utilized and it is extended on both sides by around $3 \mathrm{~cm}$ on each side. A blunt dissection is continued deep into the extraperitoneal space in the pelvis to reach the iliac vessels on the pelvic wall. The vessels are identified; the ureter is identified and separated with the medial peritoneal leaf. Anterior division of the internal iliac artery is located at $3 \mathrm{~cm}$ away from the point of division of the common iliac artery. Two ligatures are applied at this point with a delayed absorbable suture without breaching the continuity of the vessel (ligature in continuity). The femoral pulse of the same side is checked to confirm the integrity of external iliac artery.

This method has the following advantages

1. Less time consuming.
2. Peritoneal cavity not opened, so infection from contamination is avoided.

3. Structures easily identified, as intestines and omentum are not obstructing the view.

4. Handling of infectious, oedematous and friable tissues of pelvic cavity are avoided.

The author did not observe any unusual or serious complication in the present series.

\section{Complications}

1. Buttock and thigh claudication.

2. Damage to the ureter.

3. Damage to the external iliac artery leading to ischemic lower limb.

4. Damage to pelvic nerves.

5. Spinal ischemia.

6. Gangrene of the urinary bladder (rare).

\section{Conclusion}

Internal iliac artery ligation is a lifesaving procedure for a woman who is bleeding fatally due to obstetrical or gynaecological reason. This procedure can help an woman in maintaining her reproductive life with an intact uterus- specially in case of severe PPH when all other medical and conventional treatment fails. Due to its anatomical position, critically adjacent to the ureter and the iliac veins -the ligation of internal iliac artery has become a challenge to the young obstetricians. But this may be simplified by teaching the process hands on with a good anatomical briefing. This is essential for all the O\&G specialists to learn the technique of internal iliac ligation during their postgraduate course or during senior residency period. If this becomes mandatory in the post graduate curriculum, it will be a great boon to the women population in general and for the young mothers suffering from PPH in particular. The modified extraperitoneal technique of internal iliac ligation is advantageous and useful in selected cases.

Conflict of interest: None.

Disclaimer: Nil. 
The New Indian Journal of OBGYN. 2018 (January-June); 4(2)

\section{References}

1.Kelly H. Ligation of both internal iliac arteries for haemorrhage in hysterectomy for carcinoma uterus. John Hopkins Med J.1894; 5: 53-4.

2.Joshi VM. Internal iliac ligation for arresting postpartum haemorrhage. BJOG. 2007; 114(3): 356-61.

3.Desai P, Deshpande H. Postpartum haemorrhage - surgical intervention. In: Puri R, Malhotra N, Malhotra J, Shah P, editors. Operative Obstetrics and Gynaecology. 1 st ed. New Delhi: Jaypee Brothers Medical Publishers (P) Ltd; 2009. p. 218-20.

4.Bellad MB. Internal iliac artery ligation (IIAL) - An uterus/lifesaving procedure. J South Asian Federation of Obst. \& Gynaecology. 2009; 1 (2) 32-3.

\section{Prof (Dr) Hemkanta Sarma ${ }^{1}$}

${ }^{1}$ Professor \& HOD, Department of Obstetrics and Gynaecology, Jorhat Medical College, Jorhat, Assam, India 